INS-Rep-1128

December 1995

\title{
$N=2$ HETEROTIC STRING THRESHOLD CORRECTION, K3 SURFACE AND GENERALIZED KAC-MOODY SUPERALGEBRA
}

\author{
TOSHIYA KAWAI \\ Institute for Nuclear Study, University of Tokyo, \\ Midori-cho, Tanashi, Tokyo 188, Japan
}

\begin{abstract}
We study a standard-embedding $N=2$ heterotic string compactification on $K 3 \times T^{2}$ with a Wilson line turned on and perform a world-sheet calculation of string threshold correction. The result can be expressed in terms of the quantities appearing in the twoloop calculation of bosonic string. We also comment and speculate on the relevance of our result to generalized Kac-Moody superalgebra and $N=2$ heterotic-type IIA duality.
\end{abstract}




\section{Introduction}

The work of Seiberg and Witten [1,2] aroused much enthusiasm in the investigation of $N=2$ supersymmetric Yang-Mills field theories. The pursuit of analogy 3 5 in string theory has led to an exciting discovery of $N=2$ heterotic-type IIA duality [5] which is currently subject to intensive scrutiny [6 13].

To establish such duality it is important to know one-loop effects in heterotic string compactified on $K 3 \times T^{2}$, especially its moduli dependence.

One of the important quantities in probing such effects is string threshold correction to the gauge coupling. There is an extensive literature on the subject [14 27]. Roughly speaking, string threshold correction is a logarithmic sum (with coefficients of gauge charges) of masses of 'heavy modes'. Since heavy modes can be accidentally massless at some points in the moduli space we encounter logarithmic singularities at such points. There is also constraint stemming from the existence of an infinite number of light KaluzaKlein particles in the decompactification limit [19]. With this information along with the imposition of $T$-duality one can sometimes guess the moduli dependence of string threshold correction.

On the other hand there is a beautiful world-sheet calculation initiated by Dixon, Kaplunovsky and Louis [16]. The connection between the world-sheet calculation and the new supersymmetric index [28] was discovered in [20].

In a recent interesting work, Harvey and Moore [30] clarified the role played by BPS states, extended the calculation of [16] and pointed out the relevance of generalized KacMoody (super)algebras [31 34].

In this work we present the result of a world-sheet calculation similar to those in [16, 30]. We consider a standard embedding heterotic string compactified on $K 3 \times T^{2}$ with a single Wilson line turned on. Employing the formula due to Gritsenko and Nikulin [33] we show that the string threshold correction (for the portion of our interest) can be written in terms of the expression familiar in the two-loop calculation of bosonic string [35.

Interestingly, the formula of ref. [33] we will use is blessed with an interpretation of the denominator formula of a certain generalized Kac-Moody superalgebra which is a special case of the more general and geometrical constructions [34] associated with the Picard lattices of algebraic $K 3$ surfaces. Since it has been argued [36] that in string-string duality the Picard group of $K 3$ (the fiber of a $K 3$ fibration) on the type IIA side is related to the perturbatively visible gauge groups on the heterotic side, it is tempting to consider

that generalized Kac-Moody superalgebras are the right framework in which to discuss string-string duality, at least in the weak coupling regime of heterotic string. 


\section{$2 \quad K 3$ elliptic genus}

The calculation of string threshold correction involves elliptic genus [37 39]. In all likelihood, this has been known to experts for a long time although may have not been stated explicitly. In fact the appearance of elliptic genus is ubiquitous in the literature [10, 12, 16, 40]. A streamlined argument is given in ref. [30]. The relevance of elliptic genus can be intuitively understood as follows. In the case of heterotic compactification on $K 3 \times T^{2}$, string threshold correction can depend on $K 3$ only through coarse topological invariants like elliptic genus since the $K 3$ moduli fields belong to neutral hypermultiplets and hence do not affect the physics of the vector multiplets.

Recall that $(2,2)$ elliptic genus is defined by

$$
Z(\tau, z)=\operatorname{Tr}_{R, R}(-1)^{F} y^{\left(J_{L}\right)_{0}} q^{L_{0}-\frac{c}{24}} \bar{q}^{\bar{L}_{0}-\frac{c}{24}}
$$

where $q=\mathbf{e}[\tau], y=\mathbf{e}[z], \mathbf{e}[x]=\exp [2 \pi i x]$ and the trace is taken over the (Ramond, Ramond) sector. $J_{L}(z)$ is the $U(1)$ current of the left $N=2$ algebra and $c=6$ for the $K 3$ sigma model. For general properties of $N=2$ elliptic genera see refs. [42, 43], the convention of which we will use in what follows.

There are many expressions of the $K 3$ elliptic genus corresponding to many physical realizations 41 43]. For our present purpose, the following information suffices.

Due to the general properties of $(2,2)$ elliptic genus [42], it can be shown 44] that the $K 3$ elliptic genus $Z(\tau, z)$ has an expansion

$$
Z(\tau, z)=\sum_{a \in \mathbf{Z}_{\geq 0}, b \in \mathbf{Z}} c\left(4 a-b^{2}\right) q^{a} y^{b},
$$

where the coefficients $c(N)$ are some integers. As mentioned in [42], $Z(\tau, z)$ can be organized as

$$
Z(\tau, z)=h_{0}(\tau) \theta_{0,1}(\tau, 2 z)+h_{1}(\tau) \theta_{1,1}(\tau, 2 z)
$$

where

$$
\theta_{0,1}(\tau, 2 z)=\sum_{b=e v e n} q^{b^{2} / 4} y^{b}, \quad \theta_{1,1}(\tau, 2 z)=\sum_{b=o d d} q^{b^{2} / 4} y^{b}
$$

and

$$
\begin{aligned}
h_{0}(\tau) & =\sum_{N \equiv 0} c(N) q^{N / 4} \\
& =20+216 q+1616 q^{2}+8032 q^{3}+33048 q^{4}+117280 q^{5}+\cdots, \\
h_{1}(\tau) & =\sum_{N \equiv-1(\bmod 4)} c(N) q^{N / 4} \\
& =q^{-1 / 4}\left(2-128 q-1026 q^{2}-5504 q^{3}-23550 q^{4}-86400 q^{5}+\cdots\right) .
\end{aligned}
$$

\footnotetext{
${ }^{*}$ For explicit expressions, see [42]. Notice that the $h_{0}$ and $h_{1}$ there are different by a factor of $\eta(\tau)$.
} 
Note in particular that

$$
c(0)=20, \quad c(-1)=2, \quad c(N)=0 \text { if } N<-1
$$

thus

$$
Z(\tau, z)=2 y+20+2 y^{-1}+O(q) .
$$

The Witten index is given by

$$
Z(\tau, 0)=\chi(K 3)=24 .
$$

The decomposition (3) is related to the fact that an $S U(2)$ affine Lie algebra (in the present case, at level one) is contained in the $N=4$ superconformal algebra of the $K 3$ sigma model and $J_{L}(z)=2 J_{3}(z)$ where $J_{3}(z)$ is the Cartan $U(1)$ current of the affine $S U(2)$. The relevant representation theory of $N=4$ superconformal algebra can be found in [45].

\section{$3 \quad N=2$ heterotic string threshold correction with a Wilson line}

We consider a heterotic string compactification on $K 3 \times T^{2}$ with standard embedding. The massless spectrum contains an $E_{7}$ vector multiplet belonging to 133 and 10 hypermultiplets belonging to 56 of $E_{7}$. (There are other 65 gauge neutral hypermultiplets the $20 \mathrm{~K} 3$ moduli hypermultiplets and the 45 gauge bundle moduli hypermultiplets -, but these hypermultiplets do not come into our subsequent discussion.)

It is useful to review how this gauge symmetry arises from the world-sheet point of view. The gauge symmetry $E_{7}$ is entirely from the left movers and is enhanced from $S O(12) \times S U(2)$ where the $S O(12)$ symmetry is realized, in the fermionic formulation, by 12 free left gauge fermions $\lambda_{a}$ and the $S U(2)$ comes from the internal symmetry of the left $N=4$ superconformal algebra of the $K 3$ sigma model. Thus for the vector multiplet, we have

$$
133 \rightarrow\left(32_{\mathrm{c}}, 2\right)+(1,3)+(66,1)
$$

and for the hypermultiplets,

$$
56 \rightarrow\left(32_{\mathrm{s}}, 1\right)+(12,2) .
$$

The free gauge fermions $\lambda_{a}$ and the $K 3$ sigma model must be in the same periodic boundary conditions, i.e. both in the Neveu-Schwarz sectors or in the Ramond sectors. We now focus on the Ramond sector of the $K 3$ sigma model. Since the $\lambda_{a}$ in the Ramond sector realize a level one $S O(12)$ affine Lie algebra of spinor conjugacy classes, the relevant parts 
are $\left(32_{\mathbf{c}}, \mathbf{2}\right)$ for the vector multiplet and $(\mathbf{3 2}, \mathbf{1})$ for the hypermultiplets. Therefore in the decomposition (3) of the $K 3$ elliptic genus, the first term corresponds to the hypermultiplets and the second term to the vector multiplet. Note that $c(-1)$ and $c(0)$ correctly count, with a multiplicity two, the number of the vector multiplet and hypermultiplets respectively.

In addition, there are three other abelian vector multiplets associated with $S, T$ and $U$ where $S$ is the dilaton-axion, $T$ and $U$ are the Kähler and complex structure moduli of the torus $T^{2}$. The graviphoton is contained in the graviton multiplet.

We will now consider a situation where a Wilson line is turned on so as to break the above $\mathrm{SU}(2)$ gauge symmetry down to $U(1)$. This Wilson line modulus is denoted by $V$ which is a member of the vector multiplet of the unbroken $U(1)$. Thus for $V \neq 0$ the vector multiplet of $(\mathbf{3 2} \mathbf{c}, \mathbf{2})$ becomes massive while the hypermultiplets of $\left(\mathbf{3 2} \mathbf{s}_{\mathrm{s}}, \mathbf{1}\right)$ are neutral with respect to the $U(1)$ and remain massless.

We will investigate the dependence of the string threshold correction on $T, U$ and $V$. Since the Narain duality group $S O(2,3 ; \mathbf{Z})$ is isomorphic to $S p(4, \mathbf{Z})$, it is convenient to combine these parameters into a $2 \times 2$ matrix belonging to the Siegel upper half plane of genus 2:

$$
\Omega=\left(\begin{array}{cc}
T & V \\
V & U
\end{array}\right), \quad \operatorname{Im} T>0, \quad \operatorname{Im} U>0, \quad Y:=\operatorname{det} \operatorname{Im} \Omega>0 .
$$

Thus the threshold correction must be in some way expressed in terms of automorphic forms of $S p(4, \mathbf{Z})$.

We start with the integral

$$
\mathcal{I}=\int_{\mathcal{F}} \frac{d^{2} \tau}{I m \tau} \sum_{\substack{m_{1}, m_{2} \\ n_{1}, n_{2}}}\left(\sum_{b=\text { even }} q^{\frac{1}{2} p_{L}^{2}} \bar{q}^{\frac{1}{2} p_{R}^{2}} h_{0}(\tau)+\sum_{b=\text { odd }} q^{\frac{1}{2} p_{L}^{2}} \bar{q}^{\frac{1}{2} p_{R}^{2}} h_{1}(\tau)-c(0)\right)
$$

where $\mathcal{F}$ is the fundamental domain of the modular group of the world-sheet torus and

$$
\begin{aligned}
\frac{1}{2} p_{R}^{2} & =\frac{1}{4 Y}\left|m_{1} U+m_{2}+n_{1} T+n_{2}\left(T U-V^{2}\right)+b V\right|^{2} \\
\frac{1}{2}\left(p_{L}^{2}-p_{R}^{2}\right) & =\frac{1}{4} b^{2}-m_{1} n_{1}+m_{2} n_{2}
\end{aligned}
$$

with $m_{1}, m_{2}, n_{1}, n_{2}$ and $b$ running over integers. The subtraction in (13) is to remove the logarithmic singularities due to the massless hypermultiplets.

We closely follow the neat calculation done initially by Dixon, Kaplunovsky and Louis [16] and recently extended by Harvey and Moore [30]. As in these works, the calculation of $\mathcal{I}$ involves three contributions i.e. $\mathcal{I}=\mathcal{I}_{0}+\mathcal{I}_{\text {nd }}+\mathcal{I}_{\text {deg }}$.

Omitting the details the result is as follows. 
(1) zero orbit

$$
\begin{aligned}
\mathcal{I}_{0} & =\frac{Y}{U_{2}} \int_{\mathcal{F}} \frac{d^{2} \tau}{(\operatorname{Im} \tau)^{2}} Z(\tau, 0) \\
& =\frac{Y}{U_{2}} \cdot \frac{\pi}{3} \cdot 24
\end{aligned}
$$

(2) non-degenerate orbits

$$
\mathcal{I}_{n d}=-\log \prod_{k>0, l \geq 0, b \in \mathbf{Z}}|1-\mathbf{e}[k T+l U+b V]|^{4 c\left(4 k l-b^{2}\right)}
$$

(3) degenerate orbits

$$
\begin{aligned}
\mathcal{I}_{\text {deg }}= & \frac{\pi}{3} c(0) U_{2}-\log Y^{c(0)}-\log \prod_{l>0}|1-\mathbf{e}[l U]|^{4 c(0)} \\
& +\left(\gamma_{E}-1-\log \frac{8 \pi}{3 \sqrt{3}}\right) c(0)+4 \pi\left(\frac{V_{2}^{2}}{U_{2}}+V_{2}+\frac{U_{2}}{6}\right) c(-1) \\
& \quad-\log \prod_{l>0, b= \pm 1}|1-\mathbf{e}[l U+b V]|^{4 c\left(-b^{2}\right)}-\log \prod_{b=-1}|1-\mathbf{e}[b V]|^{4 c\left(-b^{2}\right)}
\end{aligned}
$$

Here $U_{2}=\operatorname{Im} U, V_{2}=\operatorname{Im} V$ and $\gamma_{E}$ is the Euler-Mascheroni constant.

Summing up the three contributions we obtain

$$
\mathcal{I}=-2 \log \kappa Y^{10}\left|\mathbf{e}[T+U+V] \prod_{(k, l, b)>0}(1-\mathbf{e}[k T+l U+b V])^{c\left(4 k l-b^{2}\right)}\right|^{2},
$$

where

$$
\kappa=\left(\frac{8 \pi}{3 \sqrt{3}} e^{1-\gamma_{E}}\right)^{10}
$$

and $(k, l, b)>0$ means that either $k>0, l \geq 0, b \in \mathbf{Z}$ or $k \geq 0, l>0, b \in \mathbf{Z}$ or $k=l=0, b<0$.

Now a remarkable formula proved by Gritsenko and Nikulin [33] shows that

$$
\chi_{10}(\Omega)=\mathbf{e}[T+U+V] \prod_{(k, l, b)>0}(1-\mathbf{e}[k T+l U+b V])^{c\left(4 k l-b^{2}\right)}
$$

where

$$
\chi_{10}(\Omega)=2^{-12} \prod_{\alpha: \text { even }}\left\{\theta_{\alpha}(\Omega, 0)\right\}^{2}
$$

is the unique cusp form of weight 10 [46]. Here $\theta_{\alpha}(\Omega, 0)$ 's are theta constants of genus 2 and the product is taken over the 10 even spin structures. Actually (22) is the "square" of the Gritsenko-Nikulin formula [33]. 
Thus finally we obtain

$$
\mathcal{I}=-2 \log \kappa Y^{10}\left|\chi_{10}(\Omega)\right|^{2} .
$$

This precise form was recently proposed indirectly in [26] by demanding the existence of an infinite number of light Kaluza-Klein particles in the decompactification limit [19] and that the only logarithmic singularity is at $V=0$ where extra massless particles appear[. The extra massless particles are related to the vector multiplets needed for symmetry restoration $U(1) \rightarrow S U(2)$ for $V=0$.

Notice that amusingly the argument of the logarithm in eq.(24) is the expression encountered in the two-loop calculation of bosonic string [35] where the double zeroes of $\chi_{10}$ at $V=0$ were related to the existence of tachyon.

\section{Generalized Kac-Moody superalgebra and string- string duality}

In ref. [34], Gritsenko and Nikulin introduced a generalized Kac-Moody superalgebra without odd real simple roots for the Picard lattice of an algebraic $K 3$ surface $X$. The Picard lattice is the lattice of $H^{1,1}(X) \cap H^{2}(X, \mathbf{Z})$ and has signature $(1, n)$ where $n \leq$ 19. The Gritsenko-Nikulin formula [33] we used in this work can be viewed [34] as the denominator formula of the generalized Kac-Moody superalgebra associated with a certain Picard lattice of signature $(1,2)$ generated by $\delta_{1}, \delta_{2}$ and $\delta_{3}$ whose intersection matrix is

$$
\left(\delta_{i} \cdot \delta_{j}\right)=\left(\begin{array}{ccc}
-2 & 2 & 2 \\
2 & -2 & 2 \\
2 & 2 & -2
\end{array}\right) .
$$

$\left\{\delta_{1}, \delta_{2}, \delta_{3}\right\}$ is the set of even real simple roots of the superalgebra. More details can be found in the original literature [33, 34]. See also related works [47, 48]. Obviously if we switch on more Wilson lines, we will have to consider more general Picard lattices and the associated superalgebra.

In ref. [30] it is claimed that the vertex operators of vector multiplets and hypermultiplets form a generalized Kac-Moody superalgebra considering vector multiplets to be even and hypermultiplets to be odd. The parity here is that of $b+1$ where $b$ is as in sect.2. The shift by 1 is due to the spectral flow between the Ramond and Neveu-Schwarz sectors. So it seems natural to consider that the algebra is that of Gritsenko and Nikulin.

The input of our calculation of string threshold correction was the $K 3$ elliptic genus. The result was related to the Picard lattice of an algebraic $K 3$ surface and its associated generalized Kac-Moody superalgebra thanks to the work of Gritsenko and Nikulin. At first

\footnotetext{
${ }^{\dagger}$ The logarithmic singularity may easily be seen from the product formula (22).
} 
sight, this is quite mysterious since mathematically remote objects are correlated. From a physical point of view, the key to solve the puzzle seems to reside in string-string duality. As mentioned in Introduction, when discussing $N=2$ heterotic-type IIA string duality, the Picard lattices of algebraic $K 3$ surfaces (the generic fibers of $K 3$ fibration Calabi-Yau threefolds) on the type IIA side are related to the perturbatively visible gauge groups on the heterotic side [36]. (Aspects of symmetry enhancement of type II string compactified

on $K 3$ have been discussed in refs. [36, 49,50].) Thus it seems quite promising to investigate string-string duality from the viewpoint of generalized Kac-Moody superalgebras. In the work of Harvey and Moore [30] the application of their results to string-string duality has been announced.

The content of this section is still speculative and apparently needs further elaboration. I hope I can return to the issue in the near future to substantiate the interpretation given here.

\section{Comments}

Even in the case of a single Wilson line that we considered in this work the string threshold correction can in principle exhibit a more complicated structure of moduli spaces [26]. It would be desirable to extend our world-sheet calculation in such a way that one can see this directly. It is also interesting to investigate the heterotic compactification with more Wilson lines turned on and to identify the Gritsenko-Nikulin superalgebras.

Another future direction would be to consider non standard-embedding $N=2$ compactifications of heterotic string. As opposed to the cases of Calabi-Yau threefolds, the $(0,2)$ elliptic genera of $K 3$ do not have much variety and are known [43].

What we have learnt in this work is restricted to the perturbative regime on the heterotic side. It would be extremely interesting to know how the picture will change non-perturbatively and how Seiberg-Witten monopole (dyon) points appear. On the type IIA side, the degenerate fibers of $K 3$ fibrations are expected to play a role [36.

In any case, it is clear that there are many things we should learn and clarify. 


\section{References}

[1] N. Seiberg and E. Witten, Nucl. Phys. B426 (1994) 19; (E) B340 (1994) 485, hepth/9407087.

[2] N. Seiberg and E. Witten, Nucl. Phys. B431 (1994) 484, hep-th/9408099.

[3] B. de Wit, V. Kaplunovsky, J. Louis and D. Lüst, Nucl. Phys. B451 (1995) 53, hep-th/9504006.

[4] I. Antoniadis, S. Ferrara, E. Gava, K.S. Narain and T.R. Taylor, Nucl. Phys. B447 (1995) 35, hep-th/9504034.

[5] S. Kachru and C. Vafa, Nucl. Phys. B450 (1995) 69, hep-th/9505105.

[6] S. Ferrara, J. A. Harvey, A. Strominger and C. Vafa, Phys. Lett. B361 (1995) 59.

[7] V. Kaplunovsky, J. Louis and S. Theisen, Phys. Lett. B357 (1995) 71, hepth/9506110.

[8] A. Klemm, W. Lerche and P. Mayr, Phys. Lett. B357 (1995) 313, hep-th/9506122.

[9] C. Vafa and E. Witten, "Dual String Pairs With N=1 And N=2 Supersymmetry In Four Dimensions," HUTP-95/A023, IASSNS-HEP-95-58, hep-th/9507050.

[10] I. Antoniadis, E. Gava, K.S. Narain and T.R. Taylor, Nucl. Phys. B455 (1995) 109, hep-th/9507115.

[11] S. Kachru, A. Klemm, W. Lerche, P. Mayr and C. Vafa, "Nonperturbative Results on the Point Particle Limit of N=2 Heterotic String," CERN-TH/95-231, HUTP95/A032, hep-th/9508155.

[12] I. Antoniadis and H. Partouche, "Exact Monodromy Group of N=2 Heterotic Superstring," CPTH-RR370.0895, hep-th/9509009.

[13] G. Aldazabal, L.E. Ibanez, A. Font and F. Quevedo, "Chains of N=2, D=4 heterotic/type II duals," CERN-TH/95-270, FTUAM-95/38, RU-95-65, UTTG-17-95, hep-th/9510093.

[14] J. Minahan, Nucl. Phys. B298 (1988) 36.

[15] V. Kaplunovsky, Nucl. Phys. B307 (1988) 145, revised version hep-th/9205070.

[16] L. J. Dixon, V. S. Kaplunovsky and J. Louis, Nucl. Phys. B355 (1991) 649. 
[17] H. Ooguri and C. Vafa, Nucl. Phys. B361 (1991) 969.

[18] S. Ferrara, C. Kounnas, D. Lüst and F. Zwirner, Nucl. Phys. B365 (1991) 431.

[19] M. Cvetic, A. Font, L.E. Ibanez, D. Lüst and F. Quevedo, Nucl. Phys. B361 (1991) 194.

[20] I. Antoniadis, E. Gava and K.S. Narain, Phys. Lett. B283 (1992) 209, hepth/9203071; Nucl. Phys. B383 (1992) 109, hep-th/9204030.

[21] I. Antoniadis, E. Gava, K.S. Narain and T.R. Taylor, Nucl. Phys B407 (1993) 706 and hep-th/9212045.

[22] I. Antoniadis and T.R. Taylor, "String loop corrections to gauge and Yukawa couplings," hep-th/9301033.

[23] P. Mayr and S. Stieberger, Nucl. Phys. B407 (1993) 725, hep-th/9303017.

[24] D. Bailin, A. Love, W.A. Sabra and S. Thomas, Phys. Lett. B320 (1994) 21, hepth/9309133; Mod. Phys. Lett. A9 (1994) 67, hep-th/9310008.

[25] G. L. Cardoso, D. Lüst and T. Mohaupt, Nucl. Phys. B450 (1995) 115, hepth/9412209.

[26] P. Mayr and S. Stieberger, Phys. Lett. B355 (1995) 107, hep-th/9504129.

[27] V. Kaplunovsky and J. Louis, Nucl. Phys. B444 (1995) 191, hep-th/9502077.

[28] S. Cecotti, P. Fendley, K. Intriligator and C. Vafa, Nucl. Phys. B386 (1992) 405, hep-th/9204102.

S. Cecotti and C. Vafa, Commun. Math. Phys. 157 (1993) 139, hep-th/9209085.

[29] M. Bershadsky, S. Cecotti, H. Ooguri and C. Vafa, Nucl. Phys. B405 (1993) 279; Commun. Math. Phys. 165 (1994) 311.

[30] J. A. Harvey and G. Moore, "Algebras, BPS States, and Strings," YCTP-P16-95, EFI-95-64, hep-th/9510182.

[31] R. Borcherds, Invent. Math. 120 (1995) 161.

[32] V. V. Nikulin, "A Lecture on Kac-Moody Lie Algebras of the Arithmetric Type," alg-geom/9412003; "Reflection Groups in Hyperbolic Spaces and the Denominator Formula for Lorentzian Kac-Moody Lie Algebras," alg-geom/9503003. 
[33] V. A. Gritsenko and V. V. Nikulin, "Siegel automorphic form corrections of some Lorentzian Kac-Moody Lie algebras," alg-geom/9504006.

[34] V. A. Gritsenko and V. V. Nikulin, "K3 surfaces, Lorentzian Kac-Moody algebras and Mirror Symmetry," alg-geom/9510008.

[35] A.A. Belavin and V.G. Knizhnik, Phys. Lett. B168 (1986) 201; Sov. Phys. JETP 64 (1986) 214. (Zh. Eksp. Teor. Fiz. 91 (1986) 364-390).

A. Belavin, V. Knizhnik, A. Morozov and A. Perelomov, Phys. Lett. B177 (1986) 324.

G. Moore, Phys. Lett. B176 (1986) 369.

A. Kato, Y. Matsuo and S. Odake, Phys. Lett. B179 (1986) 241.

[36] P. S. Aspinwall and D. R. Morrison, "String Theory on K3 Surfaces," DUK-TH-9468, IASSNS-HEP-94/23, hep-th/9404151.

P. S. Aspinwall, Phys. Lett. B357 (1995) 329, hep-th/9507012.

P.S. Aspinwall and J. Louis, "On the Ubiquity of K3 Fibrations in String Duality," CLNS-95/1369, LMU-TPW 95-16, hep-th/9510234.

P. S. Aspinwall, "Enhanced Gauge Symmetries and Calabi-Yau Threefolds," CLNS95/1375, hep-th/9511171.

[37] A.N. Schellekens and N.P. Warner, Phys. Lett. B177 (1986) 317; Nucl. Phys. B287 (1987) 317.

K. Pilch, A.N. Schellekens and N.P. Warner, Nucl. Phys. B287 (1987) 362.

[38] E. Witten, Commun. Math. Phys. 109 (1987) 525; "The Index of the Dirac Operator in Loop Space," in P.S. Landweber, ed., Elliptic Curves and Modular Forms in Algebraic Topology, (Springer-Verlag, 1988).

[39] O. Alvarez, T. Killingback, M. Mangano and P. Windey, Commun. Math. Phys. 111 (1987) 1; Nucl. Phys. B (Proc. Suppl.) 1A (1987) 189.

[40] W. Lerche, Nucl. Phys. B308 (1988) 102.

[41] T. Eguchi, H. Ooguri, A. Taormina and S.-K. Yang, Nucl. Phys. B315 (1989) 193.

[42] T. Kawai, Y. Yamada and S.-K. Yang, Nucl. Phys. B414 (1994) 191, hep-th/9306096.

[43] T. Kawai and K. Mohri, Nucl. Phys. B425 (1994) 191, hep-th/9402148.

[44] M. Eichler and D. Zagier, The Theory of Jacobi Forms, (Birkhäuser, 1985).

[45] T. Eguchi and A. Taormina, Phys. Lett. B200 (1988) 315; Phys. Lett. B210 (1988) 125. 
[46] J.-I. Igusa, Amer. J. Math. 84 (1962) 175; Amer. J. Math. 86 (1964) 392.

[47] E. Martinec, "Criticality, Catastrophes, and Compactifications," in L. Brink et. al. eds. Physics and Mathematics of Strings, V. Knizhnik memorial volume. World Scientific, 1990.

[48] I. V. Dolgachev, "Mirror symmetry for lattice polarized K3-surfaces," alg geom/9502005.

[49] E. Witten, Nucl. Phys. B443 (1995) 85.

[50] M. Bershadsky, V. Sadov and C. Vafa, "D-Strings and D-Manifolds," HUTP95/A035, IASSNS-HEP-95-77, hep-th/9510225. 